\title{
Expression level of 12-amino acid triggering receptor on myeloid cells-like transcript 1 derived peptide alleviates lipopolysaccharide-induced acute lung injury in mice
}

\author{
RUILI SHI ${ }^{1,2 *}$, JIANCHENG ZHANG ${ }^{1,3^{*}}$, ZHANG PENG $^{1}$, \\ SHIYING YUAN ${ }^{1,3}$, SUMIN GAO $^{1}$, LIN CHEN $^{1}$ and YIN YUAN ${ }^{1,3}$ \\ ${ }^{1}$ Institute of Anesthesia and Critical Care Medicine; Departments of ${ }^{2}$ Anesthesiology and ${ }^{3}$ Critical Care Medicine, \\ Union Hospital, Tongji Medical College, Huazhong University of Science and Technology, Wuhan, Hubei 430022, P.R. China
}

Received June 11, 2017; Accepted November 23, 2017

DOI: $10.3892 / \mathrm{ijmm} .2018 .3443$

\begin{abstract}
Acute lung injury (ALI) is a critical illness with a high morbidity and mortality rate due to severe inflammation in the lungs. The effects and underlying mechanism of the triggering receptor expressed on myeloid cells-1 (TREM-1)-like transcript-1-derived peptide (LR12) on ALI remain unclear. The aim of the present study was to determine whether LR12 attenuates lipopolysaccharide (LPS)-induced ALI and elucidate the mechanism underlying it. Male C57BL/6 mice were randomly assigned to three groups as follows: Sham group, LPS + scramble group and LPS + LR12 group. Normal saline (NS) or LPS was administrated by intratracheal instillation, and NS, LR12 or LR12 scramble was administered intraperitoneally $30 \mathrm{~min}$ later. The treatment was repeated every $3 \mathrm{~h}$ three times. Mice were sacrificed $24 \mathrm{~h}$ later. Pulmonary pathological changes, the lung wet/dry weight ratio, the macrophage and neutrophil counts in bronchoalveolar lavage fluid and myeloperoxidase (MPO) activity in the lung tissues were observed. The inflammatory cytokines were evaluated by enzyme-linked immunosorbent assay and lung neutrophil infiltration was detected by immunohistochemistry. Nuclear factor (NF)- $\kappa$ B p65 and TREM-1 were analyzed by western blotting, and the activation of NF- $\kappa \mathrm{B}$ was detected by electrophoretic mobility shift assay. LPS-induced pathohistological injury, edema and neutrophil infiltration were significantly alleviated by TREM-1 inhibitor, LR12. The
\end{abstract}

Correspondence to: Professor Yin Yuan, Institute of Anesthesia and Critical Care Medicine, Union Hospital, Tongji Medical College, Huazhong University of Science and Technology, 1277 Jiefang Avenue, Wuhan, Hubei 430022, P.R. China

E-mail: simiyy721@163.com

*Contributed equally

Key words: acute lung injury, triggering receptor expressed on myeloid cells-1, soluble triggering receptor expressed on myeloid cells-1, LR12, nuclear factor- $\kappa \mathrm{B}$ proinflammatory cytokines [interleukin (IL)-6, IL-1 $\beta$, tumor necrosis factor- $\alpha$ ] and chemokines (keratinocyte chemokine and monocyte chemoattractant protein-1) were significantly reduced, whereas the anti-inflammatory cytokines, IL-10 were significantly increased by LR12. LR12 was identified to significantly decrease p65 expression levels in the nucleus and inhibit the activity of $\mathrm{NF}-\kappa \mathrm{B}$. Furthermore, LR12 alleviated LPS-induced ALI by reducing the expression of TREM-1, increasing the release of soluble TREM-1 and inhibiting activation of the NF- $\mathrm{BB}$ signaling pathway.

\section{Introduction}

Acute lung injury (ALI) and acute respiratory distress syndrome (ARDS) are life-threatening conditions, the clinical manifestations of which are refractory hypoxemia and progressive dyspnea (1). Current studies indicate that the release of proinflammatory cytokines and chemokines are key factors in ALI. Inflammation is a protective defense system that acts against the invasion of pathogens $(2,3)$; however, the excessive inflammation induces tissue and organ injury (3). Excessive inflammation induced by bacterial infection performs a major role in the progression of ALI, although the exact mechanisms remain unclear (4). Blocking the progression of inflammatory cascades has been demonstrated as a valid method of mitigating ALI $(5,6)$, indicating that inflammatory blocking agents may be used to prevent and treat ALI.

In 2000, triggering receptor expressed on myeloid cells-1 (TREM-1) was first reported by Bouchon et al (7). TREM-1, a member of the immunoglobulin superfamily, is expressed on monocytes/macrophages and neutrophils $(7,8)$. TREM-1 is deemed to be an amplifier of inflammation (8). TREM-1 triggers neutrophil degranulation and oxidative burst (7,9-11), which is one of the most upregulated signaling pathways in inflammation (12). Engagement of TREM-1 with agonist monoclonal antibodies increases the secretion of tumor necrosis factor (TNF)- $\alpha$, keratinocyte chemokine (KC) and monocyte chemoattractant protein-1 (MCP-1), and inhibits the release of interleukin (IL)-10 by monocytes (13-15). Studies have demonstrated that TREM-1 is a necessary regulator of immunity and a potential therapeutic target in septic shock (16). 
Blocking TREM-1 signaling using a fusion protein or a short inhibitory peptide shows a protective effect in bacterial sepsis caused by live Escherichia coli and Pseudomonas aeruginosa, and in lipopolysaccharide (LPS)-induced shock, as well as in cecal ligation and puncture models of sepsis $(9,16-19)$. In addition, TREM-1 deficiency reduced local release of cytokines and chemokines, and delayed the influx of neutrophils (20). Furthermore, TREM-1 deficiency decreased the transepithelial migration of neutrophils into the lung (21). In other acute or chronic inflammatory diseases, the protective effects of blocking TREM-1 have been confirmed (22-25). Recently, Liu's group (26) reported the regulation of NLR family pyrin domain containing 3 (NLRP3) inflammasome activation by TREM-1, which relieves LPS-induced ALI and improves survival rate via pretreatment methods. Hence, the role of TREM-1 in LPS-induced ALI requires further investigation.

Crystallographic studies reveal that the structure between TREM-like transcript-1 (TLT-1) and TREM-1 are similar, which indicates the existence of interactions between TLT-1 and TREM-1 (27). Previous studies demonstrate that TLT-1 and LR12 behave like natural TREM-1 inhibitors, which exhibit anti-inflammatory properties by inhibiting TREM-1 signaling $(28,29)$. It has been identified that LR12 exerts protective effects in hypodynamic septic shock in pigs, and mediates inflammatory injury and cardiac remodeling following myocardial infarction in mice $(30,31)$. Furthermore, LR12 weakens endotoxin-associated clinical and biological alterations, and no obvious side effects were identified in nonhuman primates (29).

Hence, by using an LPS-induced ALI model in C57BL/6 mice, the roles and potential underlying mechanisms of TREM-1 in LPS-induced ALI were investigated in the present study.

\section{Materials and methods}

Animals. All animal experiments were approved by the Animal Care and Use Committee of Tongji Medical College of Huazhong University of Science and Technology (Wuhan, China; certification no. 2015S621). Six to eight-week old male C57BL/6 mice (weight, 20-25 g) were purchased from the animal experimental center of Wuhan University (Wuhan, China). Mice were housed (4 mice/cage) in a specific-pathogen-free room at a temperature of $22-24^{\circ} \mathrm{C}$, humidity of $60-65 \%$ and under a 12 -h light/dark cycle. The animals were allowed free access to standard laboratory chow and water, and acclimatized to the environment for 5 days before the experiments.

Introduction of LR12 and LR12 scramble. LR12 is a 12 -amino acid peptide, which is derived from TLT-1 (LQEEDAGEYGCM), and the placebo, LR12 scramble (a $\mathrm{COOH}$-terminal amidated peptide) is the corresponding scramble peptide (YQMGELCAGEED). These peptides were synthesized by Bioyeargene Biosciences Co., Ltd. (Wuhan, China). The peptides were homogeneous and with a purity of $>99 \%$, which was confirmed by mass spectrometry and analytic reversed-phase high-performance liquid chromatography. These peptides were free of endotoxin.

Experimental procedures. All the mice were divided randomly into three groups as follows: Sham, LPS + scramble and LPS +
LR12 ( $\mathrm{n}=5$ per group). The preliminary experiment identified that there was no difference in ALI between model groups treated with normal saline (NS) and LR12 scramble (data not shown). The mice were anaesthetized intraperitoneally with $90 \mathrm{mg} / \mathrm{kg}$ of $1 \%$ sodium pentobarbital (Sigma-Aldrich; Merck KGaA, Darmstadt, Germany) prior to surgery. Following successful endotracheal intubation, mice were instilled with LPS (Escherichia coli serotype O55:B5; Sigma-Aldrich, Merck $\mathrm{KGaA}$ ) at a dosage of $3 \mathrm{mg} / \mathrm{kg}$ (LPS + scramble group and LPS + LR12 group) or NS (1.5 ml/kg; sham group) (2). LR12 (5 mg/kg LPS + LR12 group), LR12 scramble (5 mg/kg; LPS + scramble group) or NS ( $5 \mathrm{ml} / \mathrm{kg}$; sham group) was administrated intraperitoneally $30 \mathrm{~min}$ after intratracheal instillation with LPS or NS (30). The treatment was repeated every $3 \mathrm{~h}$ three times.

Twenty-four hours after intratracheal instillation with LPS or NS, mice were anesthetized with an overdose of sodium pentobarbital and $1 \mathrm{ml}$ blood samples were collected from the eyeball. The blood was centrifuged at $1,000 \mathrm{x}$ g for $10 \mathrm{~min}$ at room temperature and the supernatant was stored at $-80^{\circ} \mathrm{C}$ for further experiments. The right lung was clamped at the level of the main stem bronchus. Lung tissue samples were excised and rinsed with cold phosphate-buffered saline to obtain the lung wet/dry (W/D) weight ratio and for histologic analysis, or frozen in liquid nitrogen and stored at $-80^{\circ} \mathrm{C}$ for further investigation.

Histological analysis of lung tissue samples. The right lower lobes of the lungs were embedded in paraffin wax and the sections ( 6-mm thick) were stained with hematoxylin for $10 \mathrm{~min}$ at room temperature and then with eosin for $3 \mathrm{~min}$ at room temperature. The lung injury scores were evaluated by an investigator who was blinded to the experimental design according to the published criteria (Table I): Score $=(20 \times A+14 \times B+7 \times C+7 \times D+2 \times E) /($ number of fields $\mathrm{x} 100)(32)$.

Differential leukocyte counts and pulmonary edema. Bronchoalveolar lavage fluid (BALF) was collected by bronchoalveolar lavaging with $0.4 \mathrm{ml} \mathrm{NS}$ from the left lung three times. The BALF was spun at $4^{\circ} \mathrm{C}$ for $10 \mathrm{~min}$ at $250 \mathrm{x} \mathrm{g}$. Supernatant was collected and stored at $-80^{\circ} \mathrm{C}$ for protein and cytokine detection. The cell aggregate underwent differential leukocyte counting. Total BALF cells were measured using a hematocytometer and stained with Giemsa stain solution for $30 \mathrm{~min}$ at room temperature. A total of 200 cells/slide were randomly selected to calculate the percentage of neutrophils and monocytes/macrophages in the sample under the microscope.

Pulmonary W/D weight ratio and the BALF protein concentrations were detected for the assessment of pulmonary edema. For the W/D weight ratio, the right-middle lobes were weighed as soon as they were removed. The lobe samples were placed in an oven and weighted again $24 \mathrm{~h}$ later when they were dried. BALF protein concentration was estimated using a Bicinchoninic acid Protein Assay kit (cat. no. 23227; Thermo Fisher Scientific, Inc., Waltham, MA, USA) and the procedure was performed according to the manufacturer's instructions.

Pulmonary MPO activity. Lung tissue samples were homogenized with isotonic sodium chloride to detect the MPO 
Table I. Lung injury scoring system.

\begin{tabular}{lccc}
\hline & \multicolumn{3}{c}{ Score per field } \\
\cline { 2 - 4 } Parameter & 0 & 1 & 2 \\
\hline A. Neutrophils in the alveolar space & None & $1-5$ & $>5$ \\
B. Neutrophils in the interstitial space & None & $1-5$ & $>5$ \\
C. Hyaline membranes & None & 1 & $>1$ \\
D. Proteinaceous debris filling the & None & 1 & $>1$ \\
$\quad \begin{array}{l}\text { airspaces } \\
\text { E. Alveolar septal thickening }\end{array}$ & & & \\
\hline
\end{tabular}

Score $=(20 \mathrm{xA}+14 \mathrm{xB}+7 \mathrm{xC}+7 \mathrm{xD}+2 \mathrm{xE}) /($ number of fields $\mathrm{x} 100)$.

activity. Lung tissue MPO activity was measured using the MPO kits (cat. no. A044; Nanjing Jiancheng Bioengineering Institute, Nanjing, China). Detection was conducted according to the manufacturer's instructions.

Immunohistochemistry. Following deparaffinization and repair, the sections were incubated in $3 \%$ hydrogen peroxide for $30 \mathrm{~min}$ at room temperature and blocked using $5 \%$ goat serum albumin (cat. no. SL038; Beijing Solarbio Science and Technology Co., Ltd., Beijing, China) for $20 \mathrm{~min}$ at room temperature. The sections were incubated with MPO antibody (cat. no. ab208670; 1:100; Abcam, Cambridge, MA, USA) overnight at $4^{\circ} \mathrm{C}$. Then the secondary antibody (cat. no. A25112; 1:100; Abbkine Scientific Co., Ltd., Wuhan, China) was added to the sections for $50 \mathrm{~min}$ at $4^{\circ} \mathrm{C}$. The sections were stained with 3,3'-diaminobenzidine and re-dyed with hematoxylin at room temperature. The integrated optical density was analyzed using the Image-pro Plus 6.0 software (Media Cybernetics, Inc., Rockville, MD, USA).

Immunofluorescence. Following deparaffinization, microwave-treated antigen retrieval was conducted in sodium citrate solution. Subsequently, nonspecific binding was blocked using goat serum for $30 \mathrm{~min}$. The sections were incubated with the MPO antibody (1:100) or p65 antibody (cat. no. ab16502; 1:100; Abcam) overnight at $4^{\circ} \mathrm{C}$. The secondary antibody was added for $2 \mathrm{~h}$ after washing three times with PBS. The coverslips were counterstained at room temperature with 4',6-diamidino-2-phenylindole for $5 \mathrm{~min}$ and images were acquired using a fluorescence microscope in a dark room.

ELISA. The expression levels of TNF- $\alpha$, IL-1 $\beta$, IL-6, IL-10 and $\mathrm{KC}$ in BALF were detected by using ELISA kits (cat. nos. EMC102a, EMC001b, EMC004, EMC005 and EMC104, respectively; NeoBioscience Technology Co., Shanghai, China). The expression levels of MCP-1 in BALF were evaluated using the ELISA kit (cat. no. ELM-MCP1-001; RayBiotech, Inc., Norcross, GA, USA) and sTREM-1 was analyzed with an ELISA kit (cat. no. CSB-E13848m; Cusabio Biotech Co., Ltd., Hubei, China). The intensity of the color was measured at $450 \mathrm{~nm}$ using a microplate reader (Bio-Rad Laboratories, Inc., Hercules, CA, USA) and the procedure was performed according to the manufacturer's instructions.
Western blotting. Protein was extracted from the lung tissue sample homogenates according to the manual provided by the Protein Extraction kit (cat. no. KGP150; Nanjing KeyGen Biotech Co., Ltd., Nanjing, China). The nuclear protein and cytosolic protein were separated during the procedure. Proteins were separated by electrophoresis on $10 \%$ polyacrylamide SDS gels and transferred to a polyvinylidene difluoride membrane by wet transfer $(200 \mathrm{~mA}, 1 \mathrm{~h})$. The membranes were blocked with $5 \%$ non-fat milk for $1 \mathrm{~h}$, and incubated

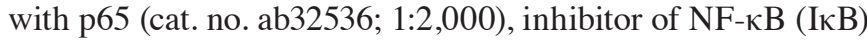
(cat. no. ab32518; 1:1,000), TREM-1(cat. no. ab104413; 1:500), $\beta$-actin (cat. no. ab8226; 1:1,000) (all Abcam) and histone H3 (cat. no. 4243; 1:500; Cell Signaling Technology, Inc., Danvers, MA, USA) individually, overnight at $4^{\circ} \mathrm{C}$. After washing three times with TBS containing $0.05 \%$ Tween-20, horse radish peroxidase-conjugated goat anti-rabbit Immunoglobulin $\mathrm{G}$ (IgG) antibodies (cat. no. SA00001-2; 1:2,000) or anti-mouse IgG antibodies (cat. no. SA00001-1; 1:2,000) (both Proteintech Group, Inc., Wuhan, China) was added at room temperature for $1 \mathrm{~h}$. Chemiluminescent detection was performed using Western Lighting Chemiluminescence Reagent (Beyotime Institute of Biotechnology, Shanghai, China). Images were scanned using a UVP imaging system and analyzed using Image J software (version 1.45s; National Institutes of Health, Bethesda, MD, USA).

Electrophoretic mobility shift assay (EMSA) for $p 65$. The protein extract was prepared using a protein extraction kit (cat. no. KGP150; Nanjing KeyGen Biotech Co., Ltd.). Each sample contained an equivalent magnitude of nuclear extract protein $(10 \mu \mathrm{g})$, and the 50 -fmol biotin-labeled, double-strand probe was incubated for $15 \mathrm{~min}$ at room temperature. The oligonucleotide probe sequence of the p65 binding site was 5'-AGTTGAGGGGACTTTCCAGGC-3'. The DNA-protein complexes were electrophoresed on a $6.5 \%$ nondenaturing polyacrylamide gel and electrotransferred for detection.

Statistical analysis. All data are presented as the mean \pm standard error of the mean. GraphPad Prism version 5.0 (GraphPad Software, Inc., La Jolla, CA, USA) was used to analyze the results. Following testing for their normal distribution (using the Kolmogorov-Smirnov test), the significance of the difference between the groups was tested by one-way ANOVA, and pairwise comparisons were performed between groups using the Newman-Keuls method. $\mathrm{P}<0.05$ was considered to indicate a statistically significant difference.

\section{Results}

LR12 attenuates pathological changes and lung injury scores in mice with LPS-induced ALI. The pulmonary construction was normal and, under light microscopy, fewer macrophages were observed in the alveolar space in the sham group (Fig. 1A). LPS treatment significantly increased the damage and hemorrhaging in the lung tissue samples. The thickness of the alveolar septal interstitium and neutrophil infiltration were significantly increased following treatment with LPS (Fig. 1A). LR12 significantly alleviated lung tissue injury when compared with the LPS + scramble group (Fig. 1A). Furthermore, the lung injury scores were assessed in parallel with the pathohistological changes (Fig. 1B). 
A

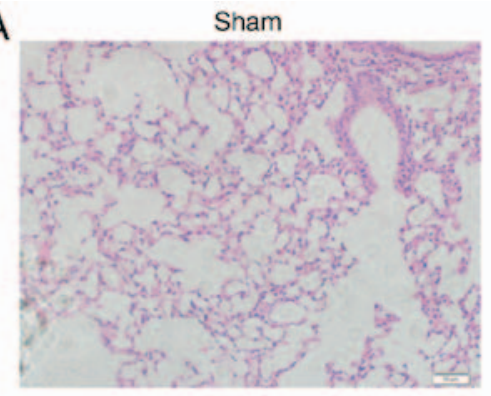

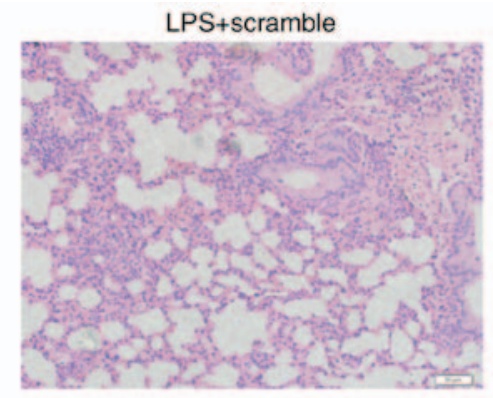

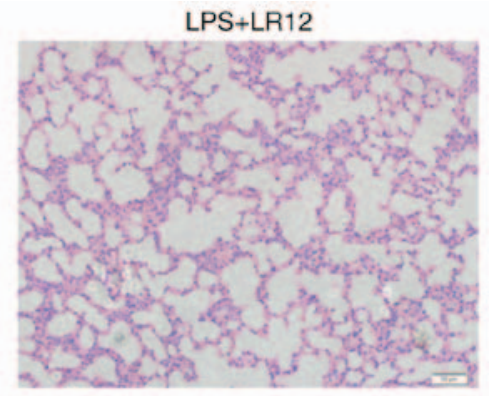

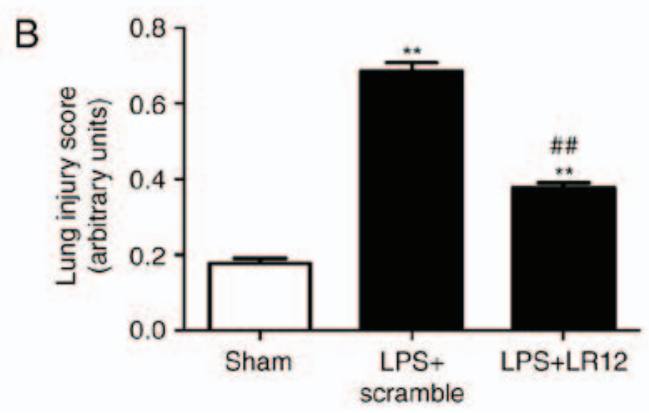

Figure 1. LR12 treatment alleviated LPS-induced ALI. (A) Morphologic changes of lung tissues (hematoxylin and eosin stain; original magnification, x200). (B) Histopathologic lung injury score. Data are presented as means \pm standard error of the mean $(\mathrm{n}=5)$. ${ }^{* *} \mathrm{P}<0.01$ vs. sham group; ${ }^{\# \#} \mathrm{P}<0.01 \mathrm{vs}$. LPS + scramble group. LPS, lipopolysaccharide; ALI, acute lung injury.
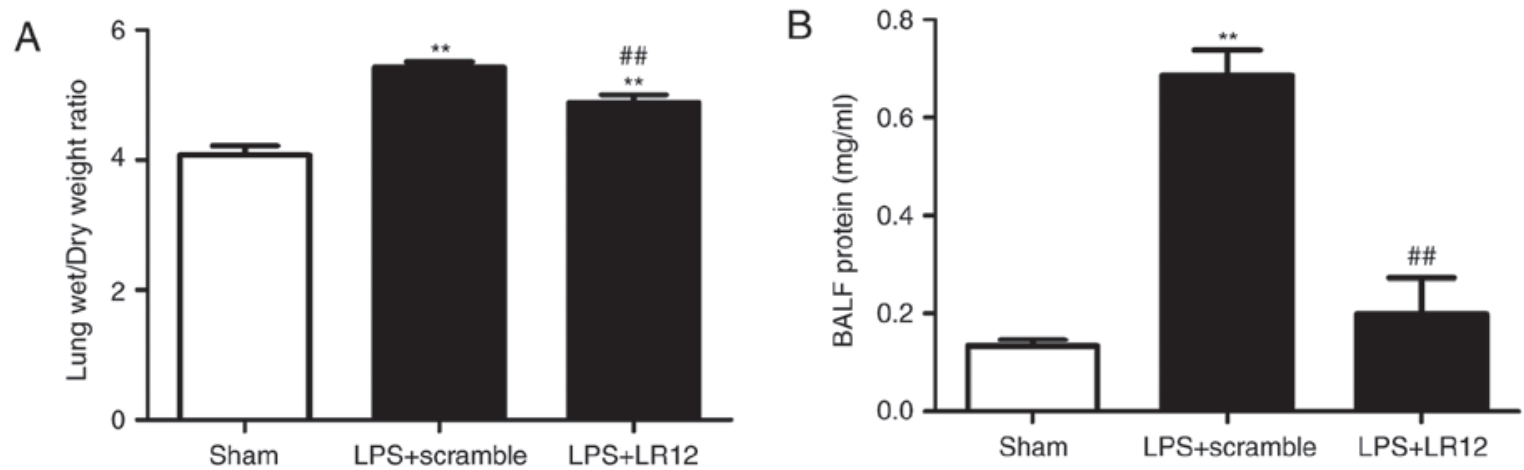

Figure 2. LR12 treatment alleviated LPS-induced permeability of ALI. (A) The lung wet/dry weight ratio. (B) The protein concentration in BALF. Data are presented as means \pm standard error of the mean $(\mathrm{n}=5) .{ }^{* *} \mathrm{P}<0.01$ vs. sham group; ${ }^{\# / *} \mathrm{P}<0.01 \mathrm{vs}$. LPS + scramble group. LPS, lipopolysaccharide; ALI, acute lung injury; BALF, bronchoalveolar lavage fluid.

LR12 attenuates the changes of lung tissue permeability in mice with $L P S$-induced ALI. The lung tissue sample W/D weight ratio and BALF protein concentration reflect the changes in lung tissue permeability. Compared with the sham group, the lung tissue sample W/D weight ratio and BALF protein concentration were significantly elevated in the LPS + scramble group, which indicated an obvious disruption in the microvascular permeability of the lung tissues. The lung tissue sample W/D weight ratio and BALF protein concentration in the LPS + LR12 group were significantly decreased compared with that in the LPS + scramble group (Fig. 2).

LR12 suppresses the variation of lung leukocyte recruitment in mice with LPS-induced ALI. Following LPS treatment, the BALF cytology changed markedly in the alveoli. Compared with that in the sham group, the numbers of total cells, macrophages and neutrophils were significantly increased in the LPS + scramble group (Fig. 3A-C).
The number of total cells and neutrophils, but not macrophages in the BALF were significantly decreased following treatment with LR12 (Fig. 3A-C).

LR12 suppresses neutrophil infiltration of lung tissues in mice with LPS-induced ALI. Immunohistochemical staining indicated that LPS treatment significantly increased the MPO-positive cells in the lung tissue samples (Fig. 4A and B). Treatment with LR12 significantly attenuated the LPS-induced significant increase of the MPO-positive cells in the lung tissue samples when compared with that in the LPS + scramble group (Fig. 4A and B). MPO activity of the lung tissue samples is an important indicator of neutrophil infiltration. The MPO activity of lung tissue samples from the LPS + LR12 group was significantly decreased when compared with that in the LPS + scramble group (Fig. 4C). The immunofluorescence staining for MPO confirmed the results (Fig. 4D). 
A

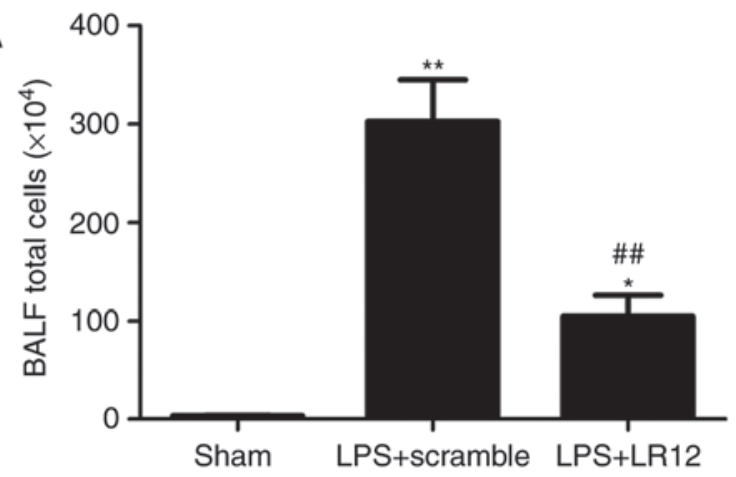

B

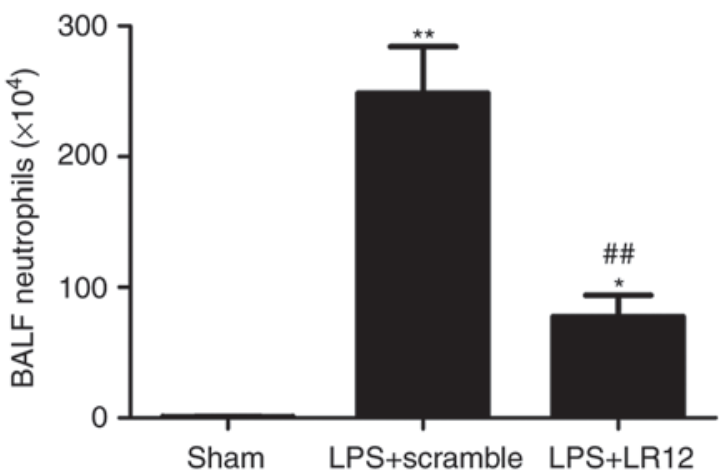

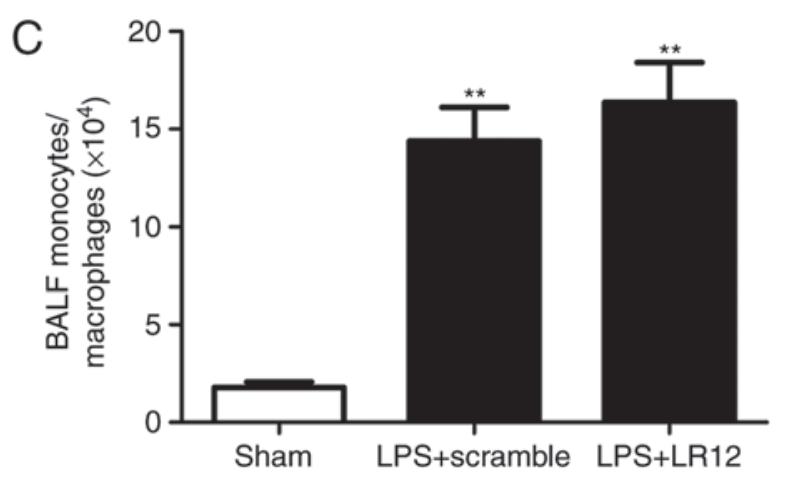

Figure 3. LR12 treatment decreased the number of total cells and neutrophils, but not macrophages in BALF. The number of (A) total cells, (B) neutrophils and $(\mathrm{C})$ monocytes/macrophages in BALF. Data are presented as means \pm standard error of the mean $(\mathrm{n}=5)$. ${ }^{*} \mathrm{P}<0.05$ and ${ }^{* *} \mathrm{P}<0.01$ vs. sham group; ${ }^{\# \#} \mathrm{P}<0.01$ vs. LPS + scramble group. BALF, bronchoalveolar lavage fluid; LPS, lipopolysaccharide.

LR12 suppresses the expression of proinflammatory cytokines and promotes the expression of anti-inflammatory cytokines in mice with LPS-induced ALI. Inflammatory cytokines are important in neutrophil recruitment and propagation of the inflammatory response. These particular inflammatory cytokines were detected in BALF using ELISA in order to investigate their effects. Compared with that in the sham group, the expression levels of proinflammatory cytokines (TNF- $\alpha$, IL-1 $\beta$, IL-6, MCP-1 and KC) were significantly increased in the LPS + scramble group (Fig. 5). Treatment with LR12 significantly attenuated the LPS-induced significant increase in proinflammatory cytokines (Fig. 5). Furthermore, the expression level of anti-inflammatory cytokine, IL-10 in the LPS + LR12 group was significantly increased when compared with that in the LPS + scramble group (Fig. 5).

LR12 inhibits $N F-\kappa B$ activation in mice with LPS-induced ALI. $\mathrm{NF}-\kappa \mathrm{B}$ existed as an inactive form combined with its inhibitor, I $\mathrm{B}$ when cells were in the resting state. The expression level of p65 in the nucleus was significantly increased with the treatment of LPS compared with that in the sham group, and administration of LR12 significantly decreased the expression level of p65 in the nucleus (Fig. 6A). The expression level of p65 and $\mathrm{I} \kappa \mathrm{B}$ in the cytoplasm was significantly increased following treatment with LR12 compared with the LPS + scramble group (Fig. 6B and C). The EMSA and immunofluorescence staining of p65 confirmed the results (Fig. 6D and E).

LR12 suppresses the expression of TREM-1 and promotes the release of STREM-1 in mice with LPS-induced ALI. The expression level of TREM-1 was significantly increased in the LPS + scramble group compared with the sham group (Fig. 7A). Following treatment with LR12, the expression level of TREM-1 in the lung tissue samples was significantly decreased (Fig. 7A). The expression levels of anti-inflammatory mediator sTREM-1 in the serum were significantly increased following treatment with LR12 compared with the LPS + scramble group (Fig. 7B).

\section{Discussion}

Inflammatory disorders, the activities and inappropriate accumulation of leukocytes and platelets, activation of uncontrollable coagulation pathways, and the changes of permeability of alveolar epithelial and endothelial barriers are the core pathophysiological characteristics of ALI/ARDS $(33,34)$. Furthermore, the glycolipid of the outer membrane of gram-negative bacteria, LPS, is a pathogenic factor of ALI/ARDS (35). Intratracheal instillation of LPS is a commendable model for ALI, which mimics gram-negative pulmonary infection in the clinical development of ALI (36).

First reported in the year 2000 (7), TREM-1 amplified the inflammatory response and contributed to the innate and adaptive immune responses (11,37-39). Under a variety of inflammatory conditions, the TREM-1 expression level was upregulated $(25,40)$ and it has been demonstrated that the expression level of TREM-1 in ALI mice was increased (41). The present study demonstrated that the expression level of TREM-1 in lung tissue samples was increased and that TREM-1 contributed significantly to mediating inflammatory cell recruitment following LPS-induced ALI. Its therapeutic modulation achieved via LR12 administration conferred protection 

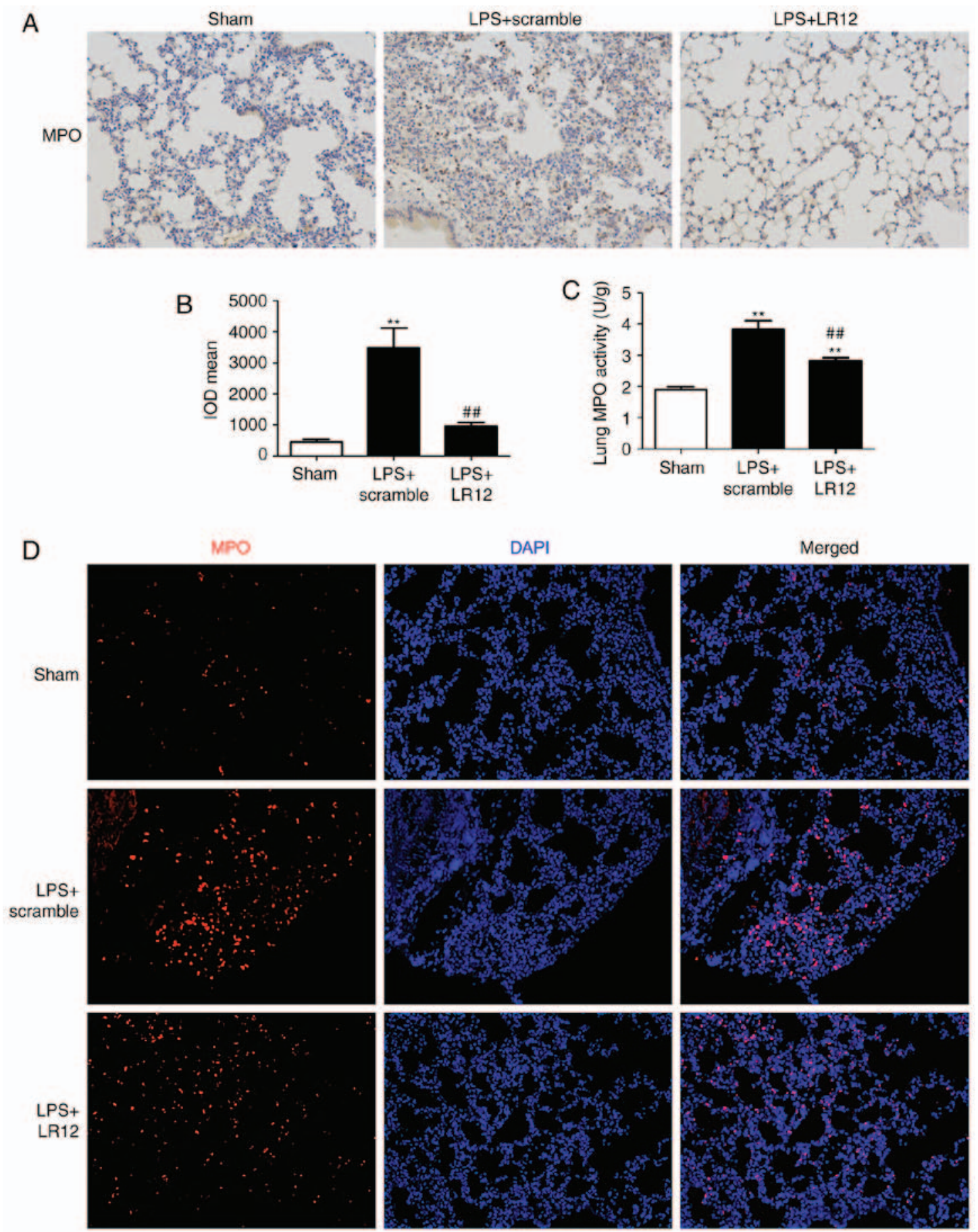

Figure 4. Infiltration and activity of neutrophils in the lung tissue samples were decreased following treatment with LR12. (A) MPO expression levels in lung tissue samples were detected by immunohistologic assay (magnification, x200). (B) MPO expression was qualified by integral optical density. (C) Lung MPO activity assay. (D) Immunofluorescent staining for MPO (magnification, $\mathrm{x} 200$ ). Data are presented as means \pm standard error of the mean $(\mathrm{n}=5) .{ }^{* *} \mathrm{P}<0.01 \mathrm{vs}$. sham group; ${ }^{\#} \mathrm{P}<0.01$ vs. LPS + scramble group. MPO, myeloperoxidase; LPS, lipopolysaccharide; DAPI, 4',6-diamidino-2-phenylindole.

in mice. LR12, a 12-amino acid sequence representative of residues 94-105, was one of the TLT-1 derived peptides (31). Previously, it was observed that TLT-1 binds and impedes TREM-1 engagement (28). Intraperitoneal injection of LR12 $(5 \mathrm{mg} / \mathrm{kg})$ was used in a study by Boufenzer et al (30), which determined a reference dose for the present study. The present study confirmed that LPS-induced ALI may be alleviated by LR12. The present data indicated that LR12 treatment significantly alleviated pathological damage, reduced pulmonary edema, decreased the infiltration and activity of neutrophils, reduced LPS-induced production of proinflammatory cytokines and chemokines (TNF- $\alpha$, IL- 6 , IL-1 $\beta$, MCP-1 and KC), and increased the release of anti-inflammatory cytokines (IL-10 and sTREM-1). Therefore, the present results indicate that LR12 confers protection against LPS-induced ALI.

$\mathrm{NF}-\kappa \mathrm{B}$, as a nuclear transcription factor, participates in LPS-induced generation of proinflammatory cytokines, chemokines and adhesion molecules (42-44). Cytokines, such as TNF- $\alpha$, IL-1 $\beta$, IL- 6 and IL-8, are transcriptionally regulated by NF- $\kappa \mathrm{B}$ in vitro (43). NF- $\mathrm{\kappa B}$ activation enhances 
A
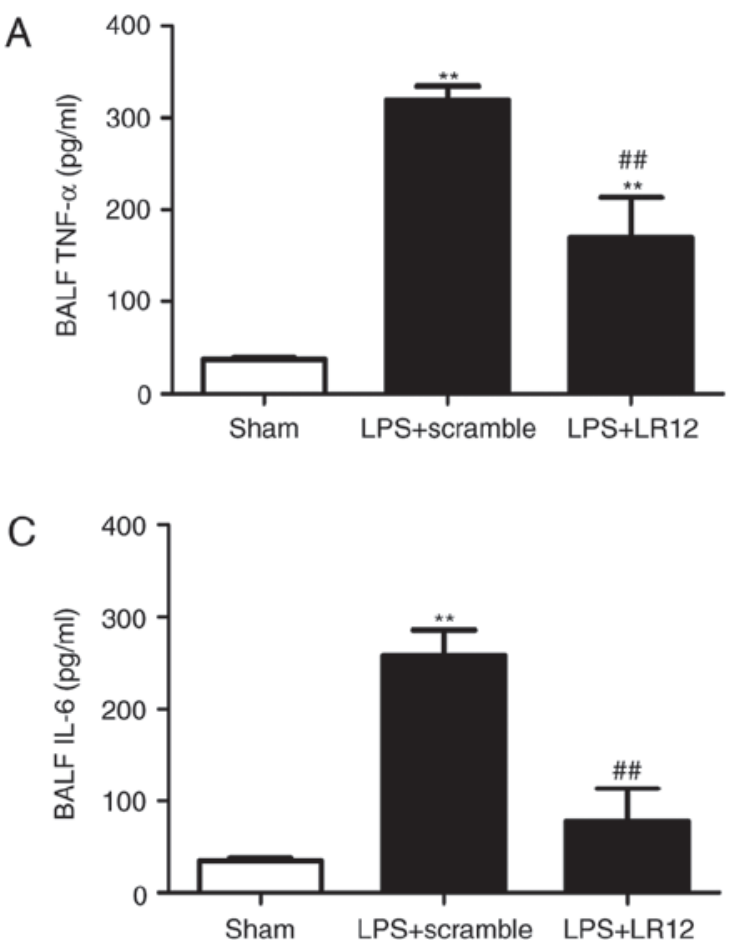

$\mathrm{E}$

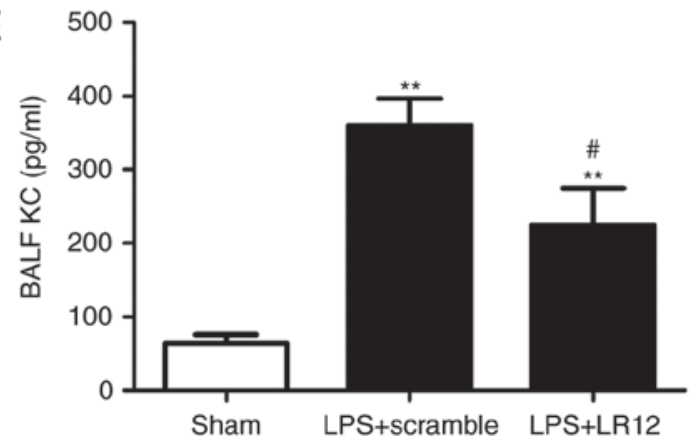

B
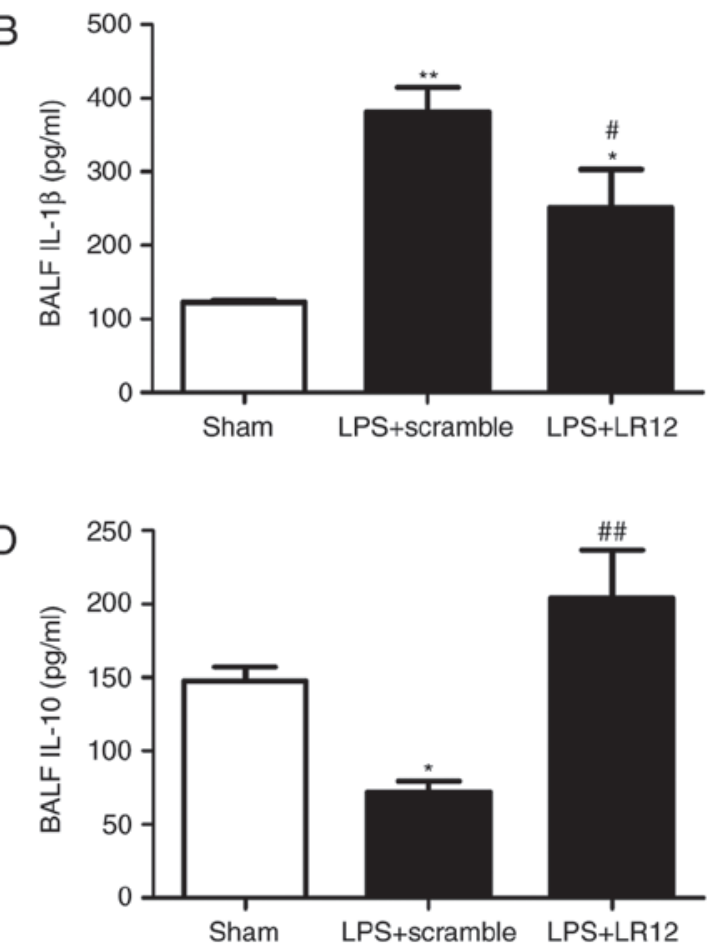

$\mathrm{F}$

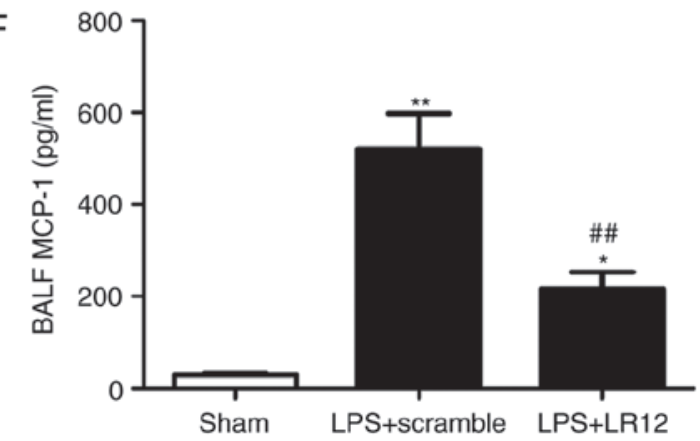

Figure 5. Treatment with LR12 significantly attenuated the LPS-induced significant increase in proinflammatory cytokines. (A) The changes of inflammatory cytokines in BALF (A) TNF- $\alpha$, (B) IL-1 $\beta$, (C) IL-6, (D) IL-10, (E) KC and (F) MCP-1. Data are presented as means \pm standard error of the mean (n=5). ${ }^{*} \mathrm{P}<0.05$ and ${ }^{* * *} \mathrm{P}<0.01$ vs. sham group; ${ }^{*} \mathrm{P}<0.05$ and ${ }^{\# \#} \mathrm{P}<0.01$ vs. LPS + scramble group. LPS, lipopolysaccharide; BALF, bronchoalveolar lavage fluid; TNF- $\alpha$, tumor necrosis factor- $\alpha$; IL, interleukin; KC, keratinocyte chemokine; MCP-1, monocyte chemoattractant protein-1.

the transcription of TNF- $\alpha$ and IL-1 $\beta$, and these cytokines activate NF- $\kappa$ B. Other mediators, such as IL-6 and IL-8, are released later and lead to more sustained elevations. These later mediators may depend largely on TNF- $\alpha$ and IL-1 $\beta$ to stimulate their production. Inflammatory stimuli, such as endotoxin, TNF- $\alpha$ and IL- $1 \beta$ stimulate the production of counterregulatory cytokines, such as IL-10, that suppress the production of proinflammatory cytokines. It was shown that IL-10 inhibits cytokine production in monocytes by blocking endotoxin-induced $N F-\kappa B$ activation (45). When bound by the I $\kappa \mathrm{B}$ family proteins, $\mathrm{NF}-\kappa \mathrm{B}$ is inactivated and sequestered in the cytoplasmic compartment. Once certain inflammatory stimuli appear, degradation of $\mathrm{I} \kappa \mathrm{B}$ family proteins is induced, which leads to the release of $\mathrm{NF}-\kappa \mathrm{B}$, followed by its translocation from the cytosol to the nucleus, where it initiates transcription of proinflammatory genes. It has been shown that NF- $\kappa \mathrm{B}$ signaling is important in mediating LPS-induced ALI $(46,47)$. Evidence has indicated that the NF- $\mathrm{BB}$ signaling pathway is involved in TREM-1-mediated inflammatory responses (48). As TREM-1 contributed to LPS-induced ALI via promotion of neutrophil infiltration, and production of proinflammatory cytokines and chemokines in the present study, it is hypothesized that TREM-1 may mediate LPS-induced ALI via activation of the NF- $\kappa \mathrm{B}$ signaling pathway. In addition, TREM-1 aggravates inflammation in ALI by activating the NLRP3 inflammasome (26). NF- $\kappa$ B inhibition leads to a dose-dependent reduction of NLRP3 protein induction by LPS, which indicates a key role for NF- $\kappa \mathrm{B}$ in priming the NLRP3 inflammasome (49). However, it was not verified by the administration of a TREM-1 inhibitor (LP17) or a NF- $\kappa$ B inhibitor (Bay 11-7082) in the present study. Future studies are required to confirm these findings.

sTREM-1, a 27-kDa glycosylated peptide that is detected in biological fluids and tissues in response to infection $(18,50,51)$, is most likely produced by cleavage of the extracellular domain from the membrane-bound form of matrix metalloproteinases (52). sTREM-1 is unable to transfer signals, but acts as a decoy receptor to prevent the binding 

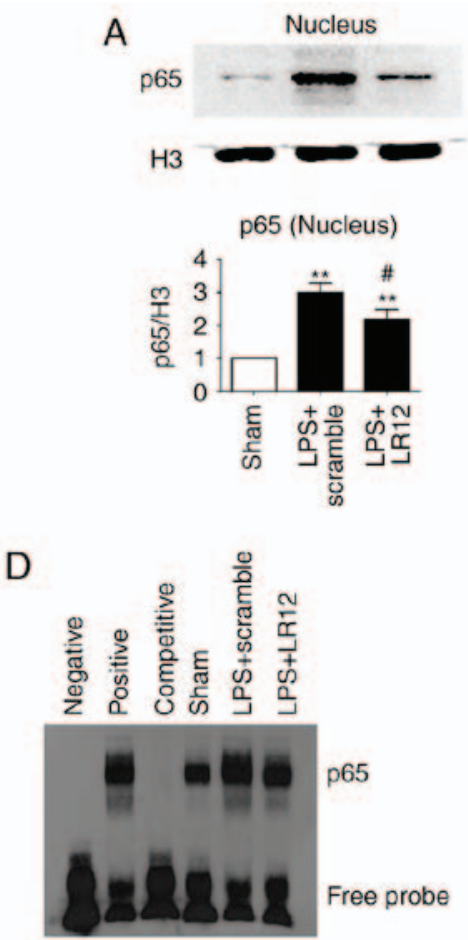
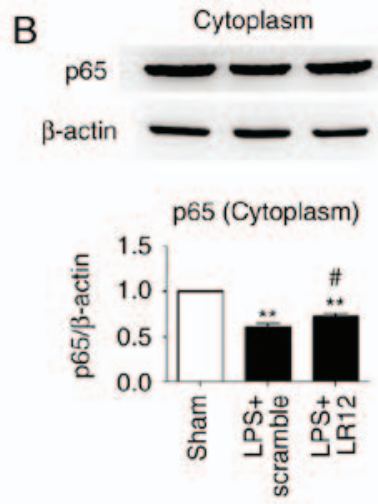
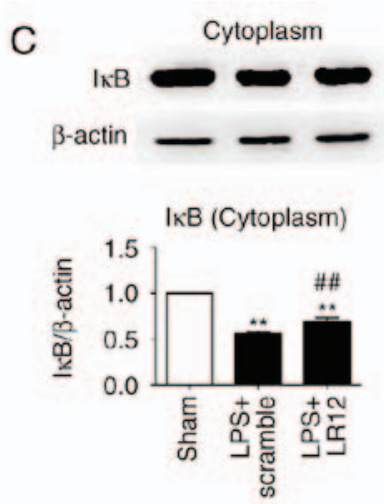

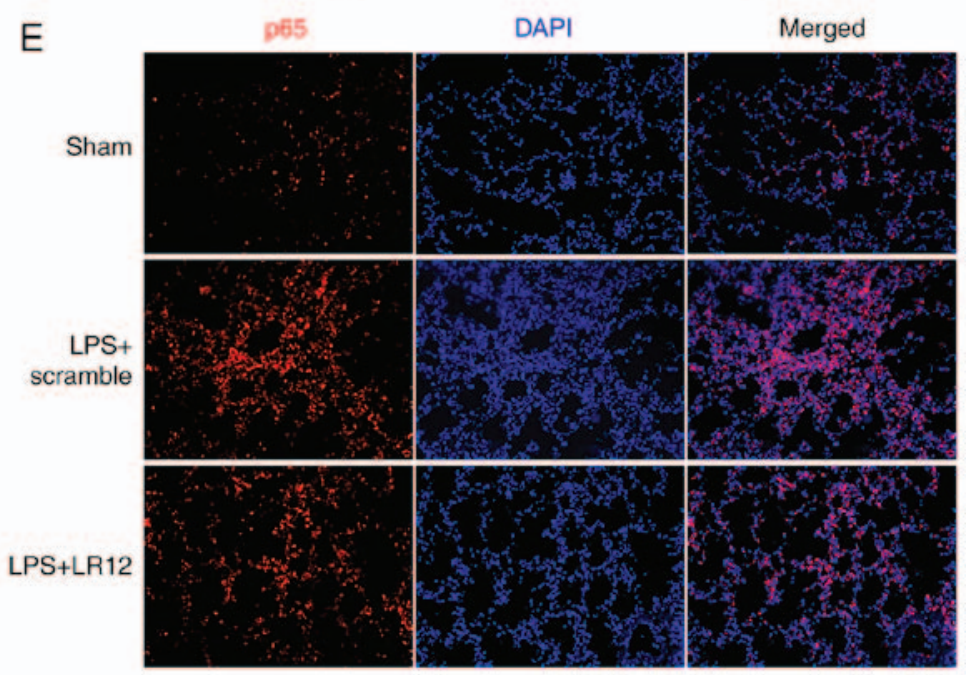

Figure 6. LR12 treatment inhibited activation of nuclear factor-кB. (A) Western blotting analysis for the expression levels of p65 in the nucleus. Histone H3 served as a loading control. (B) Western blotting analysis for the expression level of p65 in the cytoplasm. $\beta$-Actin served as a loading control. (C) Western blotting analysis for the expression of IкB in the cytoplasm. $\beta$-Actin served as a loading control. (D) p65 DNA-binding activity. The p65/DNA binding activity in nuclear extracts from lung tissue samples was detected by electrophoretic mobility shift assay. (E) Immunofluorescence staining for p65 in the nucleus (magnification, $x 200$ ). Data are presented as means \pm standard error of the mean $(\mathrm{n}=5)$. ${ }^{* * *} \mathrm{P}<0.01$ vs. sham group; ${ }^{*} \mathrm{P}<0.05$ and ${ }^{\# \#} \mathrm{P}<0.01 \mathrm{vs}$. LPS + scramble group. IкB, inhibitor of nuclear factor-кB; LPS, lipopolysaccharide; DAPI, 4',6-diamidino-2-phenylindole.
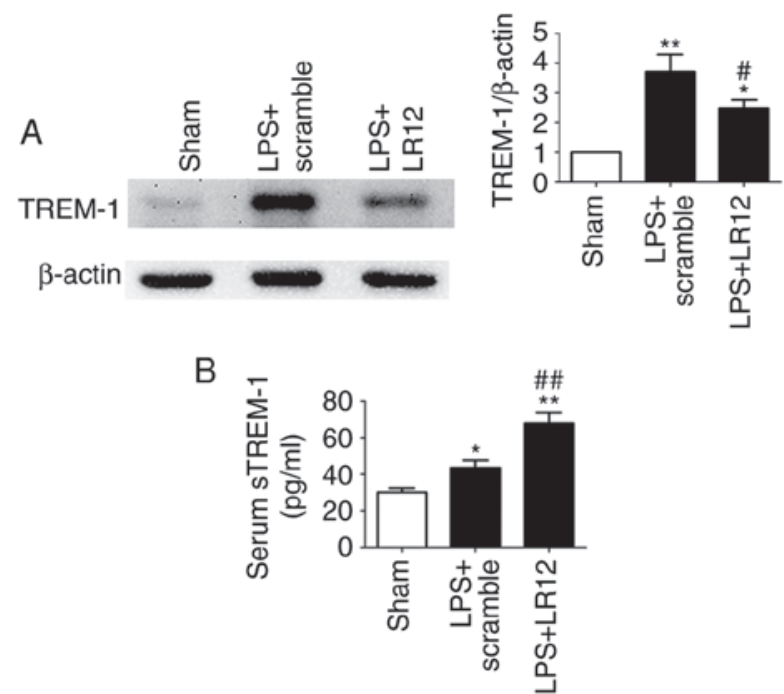

Figure 7. Expression level of TREM-1 in the lung tissue samples was decreased, whereas the expression level of sTREM-1 in the serum was increased after treatment with LR12. (A) Western blotting analysis for quantitative detection of TREM-1 expression in the lung tissue samples. (B) The expression levels of sTREM-1 in serum were detected by ELISA. Data are presented as means \pm standard error of the mean $(\mathrm{n}=5)$. ${ }^{*} \mathrm{P}<0.05$ and $^{* *} \mathrm{P}<0.01$ vs. sham group; ${ }^{*} \mathrm{P}<0.05$ and ${ }^{\# \#} \mathrm{P}<0.01$ vs. $\mathrm{LPS}+$ scramble group. TREM-1, triggering receptor expressed on myeloid cells-1; sTREM-1, soluble TREM-1; LPS, lipopolysaccharide. of its ligand to membrane-bound TREM-1, inhibit the effect of TREM-1 activation and attenuate inflammation (53). The current results revealed that the expression level of sTREM-1 increased following treatment with LR12, and it acted as an anti-inflammatory mediator, similar to IL-10 (54). The present data indicate that an increase of STREM-1 expression level after LR12 treatment could inhibit TREM-1-mediated ALI for the first time.

In conclusion, the current results clearly reveal a previously uncharacterized property of LR12, that is, the LR12-mediated anti-inflammatory effects in LPS-induced ALI. Alleviating the expression level of TREM-1, increasing the release of sTREM-1 and inhibiting activation of the NF- $\mathrm{kB}$ signaling pathway may be involved in this process. The present study provides a theoretical basis and a novel therapeutic approach for ALI/ARDS. The NF- $\kappa B$ signaling pathway was exclusively investigated in the present study, therefore, further studies are required to investigate other signaling pathways involved in this process.

\section{Acknowledgements}

The present study was supported by the National Natural Science Foundation of China (grant no. 81401568). 


\section{Competing interests}

The authors declare there is no competing interest.

\section{References}

1. Matthay MA, Ware LB and Zimmerman GA: The acute respiratory distress syndrome. J Clin Invest 122: 2731-2740, 2012

2. Gong J, Guo S, Li HB, Yuan SY, Shang Y and Yao SL: BML-111, a lipoxin receptor agonist, protects haemorrhagic shock-induced acute lung injury in rats. Resuscitation 83: 907-912, 2012.

3. Serhan CN: Resolution phase of inflammation: Novel endogenous anti-inflammatory and proresolving lipid mediators and pathways. Annu Rev Immunol 25: 101-137, 2007.

4. Tsushima K, King LS, Aggarwal NR, De Gorordo A, D'Alessio FR and Kubo K: Acute lung injury review. Intern Med 48: 621-630, 2009.

5. Shang Y, Jiang YX, Ding ZJ, Shen AL, Xu SP, Yuan SY and Yao SL: Valproic acid attenuates the multiple-organ dysfunction in a rat model of septic shock. Chin Med J (Engl) 123: 2682-2687, 2010

6. Maderna P and Godson C: Lipoxins: Resolutionary road. Br J Pharmacol 158: 947-959, 2009.

7. Bouchon A, Dietrich J and Colonna M: Cutting edge: Inflammatory responses can be triggered by TREM-1, a novel receptor expressed on neutrophils and monocytes. J Immunol 164: 4991-4995, 2000.

8. Schenk M, Bouchon A, Birrer S, Colonna M and Mueller C: Macrophages expressing triggering receptor expressed on myeloid cells-1 are underrepresented in the human intestine. J Immunol 174: 517-524, 2005.

9. Bouchon A, Facchetti F, Weigand MA and Colonna M: TREM-1 amplifies inflammation and is a crucial mediator of septic shock. Nature 410: 1103-1107, 2001

10. Colonna $\mathrm{M}$ and Facchetti F: TREM-1 (triggering receptor expressed on myeloid cells): A new player in acute inflammatory responses. J Infect Dis 187 (Suppl 2): S397-S401, 2003.

11. Dower K, Ellis DK, Saraf K, Jelinsky SA and Lin LL: Innate immune responses to TREM-1 activation: Overlap, divergence, and positive and negative cross-talk with bacterial lipopolysaccharide. J Immunol 180: 3520-3534, 2008.

12. Xiao W, Mindrinos MN, Seok J, Cuschieri J, Cuenca AG, Gao H, Hayden DL, Hennessy L, Moore EE, Minei JP, et al: A genomic storm in critically injured humans. J Exp Med 208: 2581-2590, 2011.

13. Hara H, Ishihara C, Takeuchi A, Imanishi T, Xue L, Morris SW, Inui M, Takai T, Shibuya A, Saijo S, et al: The adaptor protein CARD9 is essential for the activation of myeloid cells through ITAM-associated and Toll-like receptors. Nat Immunol 8 : 619-629, 2007.

14. Fortin CF, Lesur O and Fulop T Jr: Effects of TREM-1 activation in human neutrophils: Activation of signaling pathways recruitment into lipid rafts and association with TLR4. Int Immunol 19: 41-50, 2007.

15. Ornatowska M, Azim AC, Wang X, Christman JW, Xiao L, Joo M and Sadikot RT: Functional genomics of silencing TREM-1 on TLR4 signaling in macrophages. Am J Physiol Lung Cell Mol Physiol 293: L1377-L1384, 2007.

16. Nathan C and Ding A: TREM-1: A new regulator of innate immunity in sepsis syndrome. Nat Med 7: 530-532, 2001.

17. Wang F, Liu S, Wu S, Zhu Q, Ou G, Liu C, Wang Y, Liao Y and Sun Z: Blocking TREM-1 signaling prolongs survival of mice with Pseudomonas aeruginosa induced sepsis. Cell Immunol 272: 251-258, 2012.

18. Gibot S, Kolopp-Sarda MN, Béné MC, Bollaert PE, Lozniewski A, Mory F, Levy B and Faure GC: A soluble form of the triggering receptor expressed on myeloid cells-1 modulates the inflammatory response in murine sepsis. J Exp Med 200: $1419-1426,2004$

19. Gibot S, Buonsanti C, Massin F, Romano M, Kolopp-Sarda MN, Benigni F, Faure GC, Béné MC, Panina-Bordignon P, Passini N and Lévy B: Modulation of the triggering receptor expressed on the myeloid cell type 1 pathway in murine septic shock. Infect Immun 74: 2823-2830, 2006 .

20. Hommes TJ, Hoogendijk AJ, Dessing MC, Van't Veer C, Florquin S, Colonna M, de Vos AF and van der Poll T: Triggering receptor expressed on myeloid cells-1 (TREM-1) improves host defence in pneumococcal pneumonia. J Pathol 233: 357-367, 2014.
21. Klesney-Tait J, Keck K, Li X, Gilfillan S, Otero K, Baruah S, Meyerholz DK, Varga SM, Knudson CJ, Moninger TO, et al: Transepithelial migration of neutrophils into the lung requires TREM-1. J Clin Invest 123: 138-149, 2013.

22. Gibot S, Massin F, Alauzet C, Montemont C, Lozniewski A, Bollaert PE and Levy B: Effects of the TREM-1 pathway modulation during mesenteric ischemia-reperfusion in rats. Crit Care Med 36: 504-510, 2008.

23. Kamei K, Yasuda T, Ueda T, Qiang F, Takeyama Y and Shiozaki H: Role of triggering receptor expressed on myeloid cells-1 in experimental severe acute pancreatitis. J Hepatobiliary Pancreat Sci 17: 305-312, 2010.

24. Gibot S, Massin F, Alauzet C, Derive M, Montemont C, Collin S, Fremont $S$ and Levy B: Effects of the TREM 1 pathway modulation during hemorrhagic shock in rats. Shock 32: 633-637, 2009.

25. Schenk M, Bouchon A, Seibold F and Mueller C: TREM-1-expressing intestinal macrophages crucially amplify chronic inflammation in experimental colitis and inflammatory bowel diseases. J Clin Invest 117: 3097-3106, 2007.

26. Liu T, Zhou Y, Li P, Duan JX, Liu YP, Sun JY, Wan L, Dong L, Fang X, Jiang JX and Guan CX: Blocking triggering receptor expressed on myeloid cells-1 attenuates lipopolysaccharide-induced acute lung injury via inhibiting NLRP3 inflammasome activation. Sci Rep 6: 39473, 2016.

27. Gattis JL, Washington AV, Chisholm MM, Quigley L, Szyk A, McVicar DW and Lubkowski J: The structure of the extracellular domain of triggering receptor expressed on myeloid cells like transcript-1 and evidence for a naturally occurring soluble fragment. J Biol Chem 281: 13396-13403, 2006.

28. Derive M, Bouazza Y, Sennoun N, Marchionni S, Quigley L, Washington V, Massin F, Max JP, Ford J, Alauzet C, et al: Soluble TREM-like transcript-1 regulates leukocyte activation and controls microbial sepsis. J Immunol 188: 5585-5592, 2012.

29. Derive M, Boufenzer A and Gibot S: Attenuation of responses to endotoxin by the triggering receptor expressed on myeloid cells-1 inhibitor LR12 in nonhuman primate. Anesthesiology 120: 935-942, 2014

30. Boufenzer A, Lemarié J, Simon T, Derive M, Bouazza Y, Tran N, Maskali F, Groubatch F, Bonnin P, Bastien C, et al: TREM-1 mediates inflammatory injury and cardiac remodeling following myocardial infarction. Circ Res 116: 1772-1782, 2015.

31. Derive M, Boufenzer A, Bouazza Y, Groubatch F, Alauzet C, Barraud D, Lozniewski A, Leroy P, Tran N and Gibot S: Effects of a TREM-like transcript-1-derived peptide during hypodynamic septic shock in pigs. Shock 39: 176-182, 2013.

32. Matute-Bello G, Downey G, Moore BB, Groshong SD, Matthay MA, Slutsky AS and Kuebler WM; Acute LungInjury in Animals Study Group: An official American Thoracic Society workshop report: Features and measurements of experimental acute lung injury in animals. Am J Respir Cell Mol Biol 44: 725-738, 2011 .

33. Matthay MA and Zimmerman GA: Acute lung injury and the acute respiratory distress syndrome: Four decades of inquiry into pathogenesis and rational management. Am J Respir Cell Mol Biol 33: 319-327, 2005.

34. Matthay MA, Zimmerman GA, Esmon C, Bhattacharya J, Coller B, Doerschuk CM, Floros J, Gimbrone MA Jr, Hoffman E, Hubmayr RD, et al: Future research directions in acute lung injury: Summary of a National Heart, Lung, and Blood Institute working group. Am J Respir Crit Care Med 167: 1027-1035, 2003.

35. Moon C, Han JR, Park HJ, Hah JS and Kang JL: Synthetic RGDS peptide attenuates lipopolysaccharide-induced pulmonary inflammation by inhibiting integrin signaled MAP kinase pathways. Respir Res 10: 18, 2009.

36. Wang B, Gong X, Wan JY, Zhang L, Zhang Z, Li HZ and Min S: Resolvin D1 protects mice from LPS-induced acute lung injury. Pulm Pharmacol Ther 24: 434-441, 2011.

37. Bleharski JR, Kiessler V, Buonsanti C, Sieling PA, Stenger S, Colonna $\mathrm{M}$ and Modlin RL: A role for triggering receptor expressed on myeloid cells-1 in host defense during the early-induced and adaptive phases of the immune response. J Immunol 170: 3812-3818, 2003

38. Tessarz AS and Cerwenka A: The TREM-1/DAP12 pathway. Immunol Lett 116: 111-116, 2008.

39. Klesney-Tait J, Turnbull IR and Colonna M: The TREM receptor family and signal integration. Nat Immunol 7: 1266-1273, 2006.

40. Wang DY, Qin RY, Liu ZR, Gupta MK and Chang Q: Expression of TREM-1 mRNA in acute pancreatitis. World J Gastroenterol 10: 2744-2746, 2004. 
41. Liu N, Gu Q and Zheng YS: Expression of triggering receptor-1 in myeloid cells of mice with acute lung injury. World J Emerg Med 1: 144-148, 2010.

42. Baig MS, Zaichick SV, Mao M, de Abreu AL, Bakhshi FR, Hart PC, Saqib U, Deng J, Chatterjee S, Block ML, et al: NOS1-derived nitric oxide promotes NF-kB transcriptional activity through inhibition of suppressor of cytokine signaling-1. J Exp Med 212: 1725-1738, 2015.

43. Blackwell TS, Lancaster LH, Blackwell TR, Venkatakrishnan A and Christman JW: Differential NF-kappaB activation after intratracheal endotoxin. Am J Physiol 277: L823-L830, 1999.

44. Kang JL, Lee HW, Lee HS, Pack IS, Chong Y, Castranova V and Koh Y: Genistein prevents nuclear factor-kappa B activation and acute lung injury induced by lipopolysaccharide. Am J Respir Crit Care Med 164: 2206-2212, 2001.

45. Blackwell TS and Christman JW: The role of nuclear factor-kappaB in cytokine gene regulation. Am J Respir Cell Mol Biol 17: 3-9, 1997.

46. Zhang A, Wang $\mathrm{S}$, Zhang $\mathrm{J}$ and $\mathrm{Wu} \mathrm{H}$ : Genipin alleviates LPS-induced acute lung injury by inhibiting NF- $\kappa B$ and NLRP3 signaling pathways. Int Immunopharmacol 38: 115-119, 2016.

47. Yan C, Guan F, Shen Y, Tang H, Yuan D, Gao H and Feng X: Bigelovii A protects against lipopolysaccharide-induced acute lung injury by blocking NF- $\mathrm{BB}$ and CCAAT/Enhancer-binding protein $\delta$ pathways. Mediators Inflamm 2016: 9201604, 2016.

48. Gomez-Pina V, Martinez E, Fernández-Ruiz I, Del Fresno C, Soares-Schanoski A, Jurado T, Siliceo M, Toledano V, Fernández-Palomares R, Garcia-Rio F, et al: Role of MMPs in orchestrating inflammatory response in human monocytes via a TREM-1-PI3K-NF-кB pathway. J Leukoc Biol 91: 933-945, 2012.
49. Bauernfeind FG, Horvath G, Stutz A, Alnemri ES, MacDonald K, Speert D, Fernandes-Alnemri T, Wu J, Monks BG, Fitzgerald KA, et al: Cutting Edge: NF-kappaB activating pattern recognition and cytokine receptors license NLRP3 inflammasome activation by regulating NLRP3 expression. J Immunol 183: 787-791, 2009.

50. Knapp S, Gibot S, de Vos A, Versteeg HH, Colonna M and van der Poll T: Cutting edge: Expression patterns of surface and soluble triggering receptor expressed on myeloid cells-1 in human endotoxemia. J Immunol 173: 7131-7134, 2004.

51. Mahdy AM, Lowes DA, Galley HF, Bruce JE and Webster NR: Production of soluble triggering receptor expressed on myeloid cells by lipopolysaccharide-stimulated human neutrophils involves de novo protein synthesis. Clin Vaccine Immunol 13: 492-495, 2006

52. Gomez-Piña V, Soares-Schanoski A, Rodriguez-Rojas A, Del Fresno C, Garcia F, Vallejo-Cremades MT, Fernández-Ruiz I, Arnalich F, Fuentes-Prior P and López-Collazo E: Metalloproteinases shed TREM-1 ectodomain from lipopolysaccharide-stimulated human monocytes. J Immunol 179: 4065-4073, 2007.

53. Palazzo SJ, Simpson T and Schnapp LM: Triggering receptor expressed on myeloid cells type 1 as a potential therapeutic target in sepsis. Dimens Crit Care Nurs 31: 1-6, 2012.

54. Giamarellos-Bourboulis EJ, Zakynthinos S, Baziaka F, Papadomichelakis E, Virtzili S, Koutoukas P, Armaganidis A, Giamarellou $\mathrm{H}$ and Roussos C: Soluble triggering receptor expressed on myeloid cells 1 as an anti-inflammatory mediator in sepsis. Intensive Care Med 32: 237-243, 2006. 